\title{
Cytomegaloviral or Pneumocystis Jiroveci Pneumonia Increases Mortality in Systemic Lupus Erythematosus Patients with Pulmonary Hemorrhage: Evidence from Bronchoalveolar Lavage Fluid
}

\author{
Yi-Syuan Sun, De-Feng Huang, Fang-Chi Lin, Chih-Kai Hsu, I-Ting Sun, Shi-Chuan Chang, \\ Chang-Youh Tsai (iD, and Chien-Chih Lai
}

\begin{abstract}
Objective. To evaluate the role of cytomegaloviral or Pneumocystis jiroveci pneumonia (CMV/PJP) in systemic lupus erythematosus (SLE) patients with pulmonary hemorrhage (PH).

Methods. We retrospectively examined hospital records for 27 SLE patients with PH who received bronchoalveolar lavage fluid (BALF) analyses. Clinical profile and mortality rates were compared between groups with and without CMV/PJP. Risk factors for PH-related mortality were analyzed.

Results. Among 27 SLE patients with PH, 15 had pathogens from BALF samples, and 8 had CMV/PJP. Although CMV/PJP was treated, the RR for 90- and 180-day mortality rates of SLE patients with CMV/PJP were higher than those without these infections $(5.94,95 \%$ CI $1.44-24.48 ; 7.13,95 \%$ CI 1.81-28.06, respectively). Risk factors for 90- and 180-day mortality were presence of CMV/PJP (OR 14.2, 95\% CI 1.83-109.9; OR 25.5, 95\% CI 2.91-223.3, respectively) and use of pulse methylprednisolone for PH treatment (OR 12.0, 95\% CI 1.48-97.2; OR 8.5, 95\% CI 1.13-63.9, respectively). Factors increasing the 90-day mortality rate were duration of mechanical ventilation exceeding 14 days (OR 11.1,95\% CI 1.11-112.0) and use of aggressive immunosuppression close to PH onset (OR 7.56, 95\% CI 1.09-52.4). Three of the 7 patients receiving aggressive immunosuppression died with the presence of CMV/PJP.

Conclusion. Owing to the high prevalence of CMV/PJP and its association with mortality, routine BALF analysis is recommended for all suitable SLE patients with PH. Use of aggressive immunosuppression does not benefit SLE patients with opportunistic infections during PH attack. (First Release December 1 2018; J Rheumatol 2019;46:251-8; doi:10.3899/jrheum.180104)
\end{abstract}

Key Indexing Terms:

SYSTEMIC LUPUS ERYTHEMATOSUS CYTOMEGALOVIRUS

BRONCHOALVEOLAR LAVAGE

PULMONARY HEMORRHAGE PNEUMOCYSTIS JIROVECI PNEUMONIA

\begin{abstract}
From the Division of Allergy, Immunology, and Rheumatology, Department of Medicine, Taipei Veterans General Hospital, Taipei City; Faculty of Medicine, National Yang-Ming University, Taiwan and Institute of Clinical Medicine, National Yang-Ming University, Taipei City, Taiwan.

The study was funded partially by grants from Taipei Veterans General Hospital (V107A-005 and V107B-018). The funder has no role in study design, data collection, analysis, and interpretation of data, writing the manuscript, and the decision to submit this work for publication.

Y.S. Sun, MD, Division of Allergy, Immunology, and Rheumatology, Department of Medicine, Taipei Veterans General Hospital, and Faculty of Medicine, National Yang-Ming University; D.F. Huang, MD, Division of Allergy, Immunology, and Rheumatology, Department of Medicine, Taipei Veterans General Hospital, and Faculty of Medicine, National Yang-Ming University; F.C. Lin, MD, PhD, Department of Chest Medicine, Taipei Veterans General Hospital, and Faculty of Medicine, National Yang-Ming University; C.K. Hsu, MD, Department of Chest Medicine, Taipei Veterans General Hospital Yuli Branch; I.T. Sun, MD, Department of

Ophthalmology, E-Da Hospital, I-Shou University; S.C.Chang, MD, PhD, Professor, Department of Chest Medicine, Taipei Veterans General

Hospital, and Institute of Emergency and Critical Care Medicine, National Yang-Ming University; C.Y. Tsai, MD, PhD, Professor, Division of Allergy, Immunology, and Rheumatology, Department of Medicine, Taipei Veterans General Hospital, and Faculty of Medicine, National Yang-Ming

University; C.C. Lai, MD, Division of Allergy, Immunology, and
\end{abstract}

Rheumatology, Department of Medicine, Taipei Veterans General Hospital, and Faculty of Medicine, and Institute of Clinical Medicine, National Yang-Ming University.

Address correspondence to Dr. C.C. Lai, Division of Allergy, Immunology, and Rheumatology, Department of Medicine, Taipei Veterans General Hospital, No. 201, Sec. 2, Shipai Rd., Beitou District, Taipei City, Taiwan 11217 (R.O.C.).E-mail: cclai3@vghtpe.gov.tw

Accepted for publication August 10, 2018.

A life-threatening complication of systemic lupus erythematosus (SLE), pulmonary hemorrhage $(\mathrm{PH})$ is characterized by shortness of breath, hemosputum with decreased hemoglobulin $(\mathrm{Hgb})$ level, and new alveolar infiltrations on radiography ${ }^{1,2,3}$. Although the survival rate of $\mathrm{PH}$ in patients with SLE has improved in recent decades (overall 61\%, range $25-67 \%)^{4}$, its clinical treatment remains challenging because of the frequent presence of comorbidities, such as SLE activity and occult infections $1,5,6,7$. Prompt control of infection by pathogen-specific treatment provides a window of opportunity for immunosuppression.

Personal non-commercial use only. The Journal of Rheumatology Copyright $\odot$ 2019. All rights reserved. 
PH can be triggered by infections (virus, fungus, and bacteria) or noninfectious causes in immunocompromised hosts $^{8}$. Bronchoalveolar lavage fluid (BALF) analysis can help not only to confirm PH by hemosiderin-laden macrophages $6,7,9,10,11,12$ but also increase sensitivity and accuracy in identification of pathogens during a PH attack. Opportunistic infection, such as cytomegaloviral or Pneumocystic jiroveci pneumonia (CMV/PJP), could trigger or be associated with $\mathrm{PH}$ in patients with $\mathrm{SLE}^{13,14,15,16}$. CMV pneumonia was defined as combined pulmonary symptom and detection of CMV by viral isolation, histopathologic testing, immunohistochemical staining, or in situ hybridization and PCR from BALF ${ }^{17}$. PJP was recognized by combined pulmonary symptom and detection of $P$. jiroveci by Giemsa silver staining, toluidine blue O staining, or PCR from $\mathrm{BALF}^{18}$. BALF analysis plays an important role in the diagnosis of opportunistic infections, especially in patients with SLE under immunosuppression. Although BALF analysis may guide the precise treatment of infections in patients with SLE, this analysis is not universally performed $^{2,10,11,12}$. Further, the association of PH with CMV/PJP in patients with SLE and the outcome have not been clearly explored.

PH in patients with SLE is usually treated with high-dose glucocorticoid or pulse methylprednisolone ( $\geq 500 \mathrm{mg} /$ day), cyclophosphamide (CYC), plasmapheresis, or rituximab $(\mathrm{RTX})^{4,6,10,11,19,20}$. However, despite the use of pulse methylprednisolone or RTX for $\mathrm{PH}$ in patients with $\mathrm{SLE}^{4,6}$, the safety of these aggressive immunosuppression treatments has rarely been addressed, especially for SLE patients with concurrent opportunistic or nosocomial infections.

We conducted a retrospective study by selecting all SLE patients with $\mathrm{PH}$ for whom results of BALF analyses were available at our center. Mortality rates, risk factors of mortality, the effects of CMV/PJP, and use of aggressive immunosuppression for $\mathrm{PH}$ in patients with SLE were evaluated.

\section{MATERIALS AND METHODS}

Study population. We screened 12,274 discharge notes for patients with diagnostic codes of SLE who had been discharged from Taipei Veterans General Hospital between January 1, 2007, and January 31, 2017. Diagnostic codes for SLE were as follows: International Classification of Diseases, Ninth Revision, Clinical Modification (ICD-9-CM) code 710.0 or ICD-10-CM codes M32.0, M32.10, M32.11, M32.12, M32.13, M32.14, M32.15, M32.19, M32.8, and M32.9. We further screened these 12,274 discharge notes for patients with SLE who had undergone bronchoscopy or BAL, identifying 181 procedures. Index date of $\mathrm{PH}$ was denoted as the date that patients experienced symptoms of hemoptysis, bloody sputum, or as the date of endotracheal insertion in the patients without bloody sputum initially. In total, $33 \mathrm{BAL}$ procedures were performed in 32 patients (1 patient received a second $\mathrm{BAL}$ for the recurrent $\mathrm{PH}$ ). For the 1 patient with recurrent episodes of $\mathrm{PH}$, only the initial event was enrolled. All enrolled patients met the 1997 American College of Rheumatology SLE Classification Criteria or the 2012 Systemic Lupus International Collaborating Clinics Revised Classification Criteria ${ }^{21,22}$.

Clinical diagnosis of $\mathrm{PH}$ was verified by the presence of all of the following criteria: (1) newly formed alveolar patches in chest radiograph or chest computed tomography; (2) decrease in Hgb level exceeding $1.5 \mathrm{~g} / \mathrm{dl}$; and (3) clinical symptoms, such as shortness of breath, bloody sputum, or desaturation ${ }^{1,2,3}$. Five patients were excluded from the study because of warfarin overdose $(n=1)$, not fulfilling the clinical diagnostic criteria for $\mathrm{PH}(\mathrm{n}=2)$, or receiving BAL procedures before $\mathrm{PH}(\mathrm{n}=2)$. Finally, 27 SLE patients with $\mathrm{PH}$ were enrolled in this study (Supplementary Figure 1, available with the online version of this article). This study was approved by the Institutional Review Board of Taipei Veterans General Hospital (IRB-TPEVGH No. 2017-03-010C).

BALF analysis, followup period, and outcomes. Presence of CMV/PJP is highly associated with $\mathrm{PH}^{13,16}$. We divided SLE patients into 2 groups according to the status of these 2 infections, as confirmed by clinical presentation and BALF analysis. CMV pneumonia was determined by either positive finding of CMV on PCR from BALF or CMV virus isolation from BALF. PJP was defined by a positive finding on Giemsa silver or toluidine blue $\mathrm{O}$ staining from BALF.

Patients were followed for 180 days after index date of PH. Primary endpoint was duration of survival. Outcomes were mortality rates and RR of mortality within 90 and 180 days after index date of $\mathrm{PH}$, comparing with patients without CMV/PJP.

Clinical, laboratory, and microbiological variables. Baseline characteristics, including clinical status of SLE, medications and treatment for $\mathrm{PH}$, were collected. The Systemic Lupus Erythematosus Disease Activity Index 2000 (SLEDAI-2K) score was evaluated at admission. Hgb level was recorded as (1) the lowest Hgb level within 1 week before BAL procedure if mechanical ventilation was not required, or (2) the $\mathrm{Hgb}$ level within $24 \mathrm{~h}$ of endotracheal tube insertion. Levels of complement, antibodies against dsDNA, and serum IgG were collected within 1 month before the index date of PH. Autoantibodies against Smith, RNP, and antineutrophilic cytoplasmic antigen were documented within 1 year before $\mathrm{PH}$. Alanine transaminase was recorded as highest value within 2 weeks after index date. Dosages of steroid and disease-modifying antirheumatic drugs were recorded from the most recent outpatient department medical records before PH. For patients receiving mechanical ventilation, scores on the Acute Physiology and Chronic Health Evaluation (APACHE) $\mathrm{II}^{23}$, and the Sequential Organ Failure Assessment $(\mathrm{SOFA})^{24}$ were recorded on the date of mechanical ventilation.

Risk factors for study endpoints. PH significantly increases the risk of mortality for patients with SLE, which may be related to global SLE disease activity and/or presence of uncontrolled opportunistic infection secondary to immunosuppression ${ }^{7,25}$. Immunosuppressive therapy for patients with SLE may increase risks of infection and PH-related mortality ${ }^{6}$. Thus, we defined the use of aggressive immunosuppression close to $\mathrm{PH}$ onset as the use of (1) pulse methylprednisolone at a dose $\geq 500 \mathrm{mg} /$ day within 1 month before or after PH, and (2) RTX within 6 months before PH. Other clinical variables were analyzed for their effects on study endpoints: presence of CMV pneumonia or PJP, SLEDAI-2K, dose of systemic glucocorticoid equivalent to prednisolone dose $>7.5 \mathrm{mg} /$ day, hypogammaglobulinemia (defined as serum IgG $<751 \mathrm{mg} / \mathrm{dl}$ ), bacteremia, presence of pathogens in BALF, duration of mechanical ventilation $>14$ days, and treatment strategies for $\mathrm{PH}$.

Statistical analyses. Fisher's exact or Mann-Whitney U tests were used as appropriate to compare variables between groups and mortality rates during the study period. The Kaplan-Meier method with the log-rank test was used for survival analysis in patients with $\mathrm{PH}$ according to the status of CMV/PJP Binary logistic regression was conducted to analyze risk factors for mortality. Multivariable tests were performed by selecting factors with $\mathrm{p} \leq 0.05$ in univariate tests in a forward stepwise approach. All statistical analyses were performed using SPSS software version 20 (SPSS Inc.).

\section{RESULTS}

Baseline characteristic of SLE patients with PH. This study included 27 SLE patients (26 women) with PH. The median

Personal non-commercial use only. The Journal of Rheumatology Copyright (C) 2019. All rights reserved 
age of patients was 33.4 years, and the SLE disease activity was high (median SLEDAI-2K score: 12). Most patients had an SLE duration exceeding 3 years $(n=19)$ and a history of lupus nephritis $(\mathrm{n}=24)$. Laboratory data and background medications are described in Table 1. All patients had SLE and $\mathrm{PH}$. There were 22 patients (81.5\%) with $\mathrm{PH}$ and respiratory failure, who were supported with mechanical ventilation for a median duration of 16.3 days. BAL procedures were performed within a mean of 6.6 days after PH onset. All patients were divided into 2 groups: those who were either positive for CMV/PJP and those who were negative for both. There was no difference between the groups for most baseline variables, including demographic data, SLE disease activity, and background medications. SLE patients with CMV/PJP were more likely to be treated aggressively, including intravenous infusions of Ig and pulse methylprednisolone for $\mathrm{PH}$ (both $\mathrm{p}=0.044$ ).

Microbiological profiles of BALF. Pneumonia was frequently found in SLE patients with PH. Fifteen patients (55.6\%) had concomitant pneumonia, including bacterial, fungal, or

Table 1. Baseline characteristics of pulmonary hemorrhage of patients with SLE.

\begin{tabular}{|c|c|c|}
\hline Variables & CMV-/PJP-, n = 19 & $\mathrm{CMV}$ or $\mathrm{PJP}+, \mathrm{n}=8$ \\
\hline Age at hospitalization, yrs & $33.2(28.3-43.7)$ & $34.2(32.7-56.0)$ \\
\hline Female sex & $18(94.7)$ & $8(100)$ \\
\hline Duration of SLE $>3$ yrs & $14(73.7)$ & $5(62.5)$ \\
\hline History of lupus nephritis & $17(89.5)$ & $7(87.5)$ \\
\hline SLEDAI-2K score & $12(7-19)$ & $11.5(3.3-20.3)$ \\
\hline BAL procedure after $\mathrm{PH}$ onset, days & $5(3-6)$ & $5.5(0.75-20)$ \\
\hline Hemosiderin-laden macrophage in BALF & $8(50)$ & $5(71.4)$ \\
\hline \multicolumn{3}{|l|}{ Laboratory data } \\
\hline WBC count, $10^{3} / \mu 1$ & $8.29(4.8-11.3)$ & $6.45(1.58-15.42)$ \\
\hline Lymphocyte count, $10^{3} / \mu 1$ & $0.58(0.35-1.00)$ & $0.29(0.19-0.69)$ \\
\hline Hemoglobin, $g / 1$ & $7.2(6.0-8.0)$ & $7.1(6.6-7.9)$ \\
\hline Platelet count, $10^{9} / 1$ & $87(57-171)$ & $134(92-190)$ \\
\hline Serum $\mathrm{IgG}, \mathrm{mg} / \mathrm{dl}$ & $934(699-1530)$ & $874(565-874)$ \\
\hline Serum albumin, $\mathrm{g} / \mathrm{dl}$ & $2.8(2.5-3.0)$ & $2.7(2.1-3.0)$ \\
\hline Serum creatinine, $\mathrm{mg} / \mathrm{dl}$ & $2.27(1.21-4.25)$ & $2.51(1.83-4.67)$ \\
\hline Alanine aminotransferase, $\mathrm{U} / 1$ & $42(26-99)$ & $49(35-62)$ \\
\hline C-reactive protein, $\mathrm{mg} / \mathrm{dl}$ & $2.17(0.68-5.29)$ & $3.47(0.16-20.11)$ \\
\hline $\mathrm{C} 3, \mathrm{mg} / \mathrm{dl}$ & $47.6(34.0-67.0)$ & $59.4(46.3-79.4)$ \\
\hline $\mathrm{C} 4, \mathrm{mg} / \mathrm{dl}$ & $12.4(5.1-17.8)$ & $15.1(9.4-28.7)$ \\
\hline Anti-dsDNA antibody positivity & $9(47.4)$ & $3(37.5)$ \\
\hline Anti-Sm antibody positivity & $2(28.6)$ & $1(20)$ \\
\hline Anti-RNP antibody positivity & $3(42.9)$ & $3(60)$ \\
\hline \multicolumn{3}{|l|}{ Background medications } \\
\hline Prednisolone equivalent, $\mathrm{mg}$ /day & $10(5-15)$ & $17.5(11.3-23.8)$ \\
\hline Hydroxychloroquine & $4(21.1)$ & $2(25)$ \\
\hline Azathioprine & $4(21.1)$ & $2(25)$ \\
\hline Mycophenolate & $3(15.8)$ & $2(25)$ \\
\hline Calcineurin inhibitors & $2(10.5)$ & $1(12.5)$ \\
\hline Cyclophosphamide* & $3(15.8)$ & 0 \\
\hline Rituximab** & $1(5.3)$ & $1(12.5)$ \\
\hline Ventilator usage & $15(78.9)$ & $7(87.5)$ \\
\hline APACHE II score ${ }^{\dagger}$ & $26(22-27)$ & $24(24-28)$ \\
\hline SOFA score ${ }^{\dagger}$ & $9(8-11)$ & $8(6-12)$ \\
\hline ECMO usage $^{\dagger}$ & 0 & $2(33.3)$ \\
\hline \multicolumn{3}{|l|}{ Treatment for PH } \\
\hline Plasmapheresis & $11(57.9)$ & $4(50)$ \\
\hline IVIG & $2(10.5)$ & $4(50)^{\ddagger}$ \\
\hline IV pulse methylprednisolone & $2(10.5)$ & $4(50)^{\ddagger}$ \\
\hline Cyclophosphamide & 0 & $1(12.5)$ \\
\hline
\end{tabular}

Values are presented as median (IQR) or $\mathrm{n}(\%) .{ }^{*}$ Within 3 months or ${ }^{* *} 6$ months before pulmonary hemorrhage. $\dagger$ Only for patients ever supported with mechanical ventilation. ${ }^{\ddagger} \mathrm{p}<0.05$ vs patients without CMV pneumonia or PJP (CMV-/PJP-). SLE: systemic lupus erythematosus; APACHE II: Acute Physiology and Chronic Health Evaluation II; BAL: bronchoalveolar lavage; BALF: BAL fluid; CMV: cytomegalovirus pneumonia; ECMO: extracorporeal membrane oxygenation; IVIG: intravenous Ig; PH: pulmonary hemorrhage; PJP: Pneumocystic jiroveci pneumonia; SLEDAI-2K: SLE Disease Activity Index 2000; SOFA: sequential organ failure assessment; WBC: white blood cell; IQR: interquartile range. 
CMV/PJP, identified from BALF. Five of the 15 patients (18.5\%) had CMV/PJP infection without other associated bacterial or fungal pneumonia. In 9 of 27 patients (33.3\%), BALF cultures yielded bacteria, including Acinetobacter baumanii complex $(\mathrm{n}=4)$, Stenotrophomonas maltophilia $(\mathrm{n}=2)$, Pseudomonas species $(\mathrm{n}=1)$, Prevotella species $(\mathrm{n}=1)$, Klebsiella pneumoniae $(\mathrm{n}=1)$, or viridans strepto$\operatorname{cocci}(\mathrm{n}=1)$. Three patients had combined bacteria or fungal infection from BALF, two had combined A. baumanii complex and S. maltophilia infections, and one had combined Candida parapsilosis and Trichosporon asahii infections. We used antibiotic treatment for all patients with suspected infections and further adjusted the regimen according to the sensitivity reports from BALF. For patients with CMV/PJP, intravenous gancyclovir was used for CMV and trimethoprim-sulfamethoxazole for $P$. jiroveci.

Survival analysis of SLE patients with PH. During the followup period, the median duration of survival for SLE patients with CMV/PJP identified from BALF was significantly shorter than that of SLE patients without these infections (46 vs 180 days, $\mathrm{p}<0.001$ ). Kaplan-Meier analysis indicated an unfavorable outcome of SLE patients with CMV/PJP during PH attacks compared to SLE patients without CMV/PJP (Figure 1). The 90- and 180-day mortality rates were significantly higher among SLE patients with CMV/PJP among all enrolled patients and among patients supported with mechanical ventilation (Table 2). Compared to patients without CMV/PJP, the RR $(95 \% \mathrm{CI})$ for mortality was significantly higher for patients either positive of CMV/PJP identified from BALF (90-day mortality: RR 5.94, 95\% CI 1.44-24.48, $\mathrm{p}=0.0137$; 180-day mortality: RR 7.13 $95 \%$ CI $1.81-28.06, p=0.005)$. In mechanically ventilated patients, the RR for patients with CMV/PJP remained high in comparison with patients without CMV/PJP (both were RR 5.36, 95\% CI 1.36-21.14, $\mathrm{p}=0.0165$; Table 2). In 8 patients with CMV/PJP, 3 patients had concomitant CMV and PJP, and all 3 patients died within 23-44 days after $\mathrm{PH}$ onset.

Risk factors for PH-related mortality. Presumptive risks factors for short-term (90-day) and longterm (180-day) mortality for SLE patients with PH were analyzed and are summarized in Table 3. CMV/PJP in BALF and pulse methylprednisolone for treatment of $\mathrm{PH}$ were associated with short- and longterm PH-related mortality rates in patients with SLE (Table 3). Prolonged mechanical ventilation (> 14 days) and aggressive immunosuppression close to $\mathrm{PH}$ onset were associated with short-term PH-related mortality in

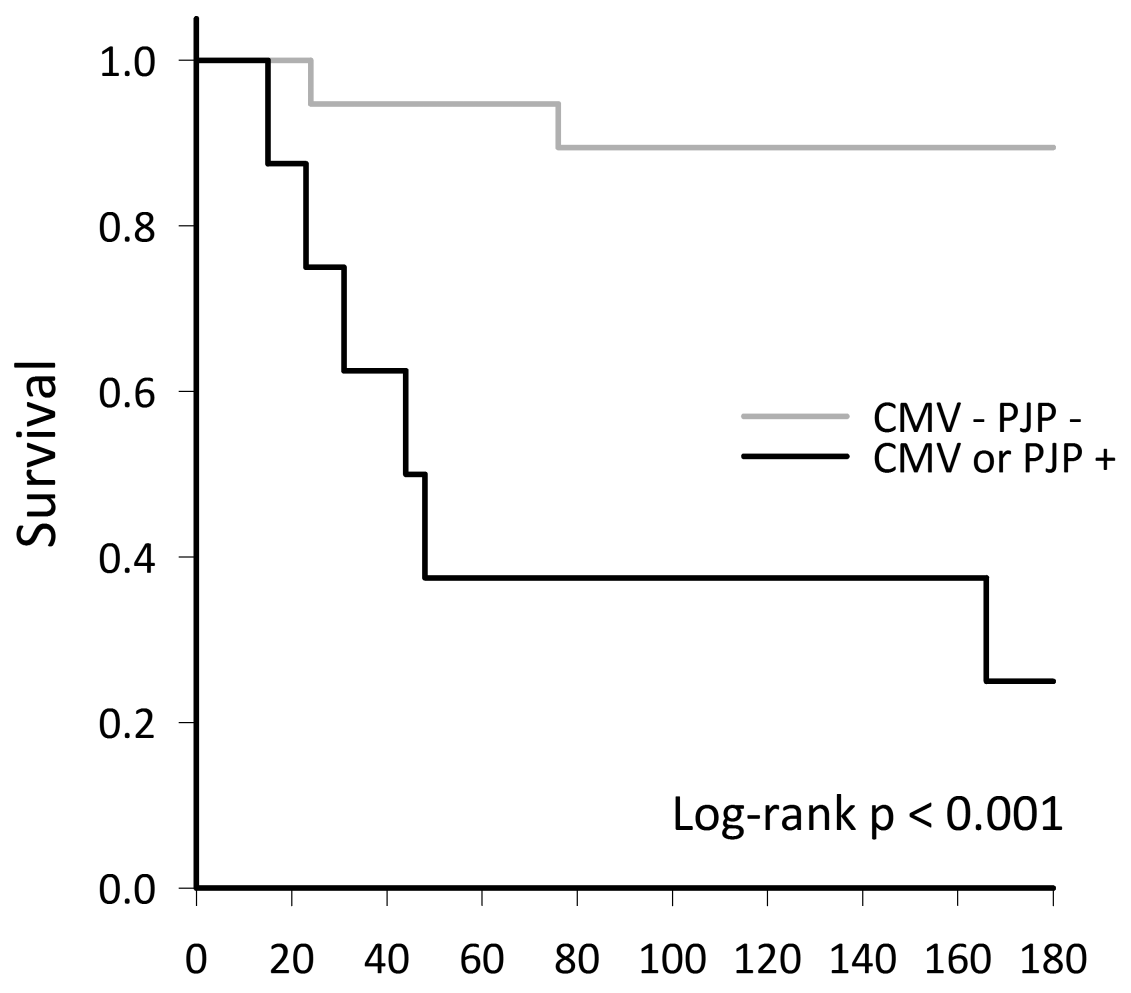

$\begin{array}{lccccccccc}\text { Number at risk } & & & & & & & & & \\ \text { CMV - PJP - 19 } & 19 & 18 & 18 & 17 & 17 & 17 & 17 & 17 & 17 \\ \text { CMV or PJP + 8 } & 7 & 4 & 3 & 3 & 3 & 3 & 3 & 2 & 2\end{array}$

Figure 1. Survival analysis of pulmonary hemorrhage in patients with systemic lupus erythematosus with or without CMV or PJP. CMV: cytomegaloviral pneumonia; PJP: Pneumocystic jiroveci pneumonia.

Personal non-commercial use only. The Journal of Rheumatology Copyright $\odot$ 2019. All rights reserved. 
Table 2. Mortality rates of PH in patients with SLE according to CMV or PJP identified from BALF.

\begin{tabular}{|c|c|c|c|c|c|c|c|}
\hline Subgroup & $\mathrm{N}$ & 90-day Mortality, n (\%) & $\mathrm{RR}(95 \% \mathrm{CI})$ & $\mathrm{p}$ & 180-day Mortality, n (\%) & $\mathrm{RR}(95 \% \mathrm{CI})$ & $\mathrm{p}$ \\
\hline All patients & 27 & $7(25.9)$ & - & - & $8(29.6)$ & - & - \\
\hline CMV (-) / PJP (-) & 19 & $2(10.5)$ & Ref & Ref & $2(10.5)$ & Ref & Ref \\
\hline CMV (+) / PJP (-) & 4 & $2(50)$ & $4.75(0.92-24.41)$ & 0.0621 & $3(75)$ & $7.13(1.71-29.71)$ & 0.0070 \\
\hline CMV (-) / PJP (+) & 1 & $0(0)$ & $2.00(0.14-28.76)$ & 0.6103 & $0(0)$ & $2.00(0.14-28.76)$ & 0.6103 \\
\hline CMV (+) / PJP (+) & 3 & $3(100)$ & $9.50(2.56-35.24)$ & 0.0008 & $3(100)$ & $9.50(2.56-35.24)$ & 0.0008 \\
\hline $\operatorname{CMV}(+)$ or PJP $(+)$ & 7 & $5(71.4)$ & $5.36(1.36-21.14)$ & 0.0165 & $5(71.4)$ & $5.36(1.36-21.14)$ & 0.0165 \\
\hline CMV (+) / PJP (-) & 3 & $2(66.7)$ & $5.00(1.10-22.82)$ & 0.0377 & $2(66.7)$ & $5.00(1.10-22.82)$ & 0.0377 \\
\hline CMV (-) / PJP (+) & 1 & $0(0)$ & $1.60(0.11-22.80)$ & 0.7288 & $0(0)$ & $1.60(0.11-22.80)$ & 0.7288 \\
\hline $\mathrm{CMV}(+) / \mathrm{PJP}(+)$ & 3 & $3(100)$ & $7.50(2.06-27.25)$ & 0.0022 & $3(100)$ & $7.50(2.06-27.25)$ & 0.0022 \\
\hline
\end{tabular}

SLE: systemic lupus erythematosus; BALF: bronchoalveolar lavage fluid; CMV: cytomegalovirus pneumonia; MV: mechanical ventilation; PH: pulmonary hemorrhage; PJP: Pneumocystic jiroveci pneumonia.

Table 3. Risk factor analysis for 90-day and 180-day mortality rates in SLE patients with pulmonary hemorrhage.

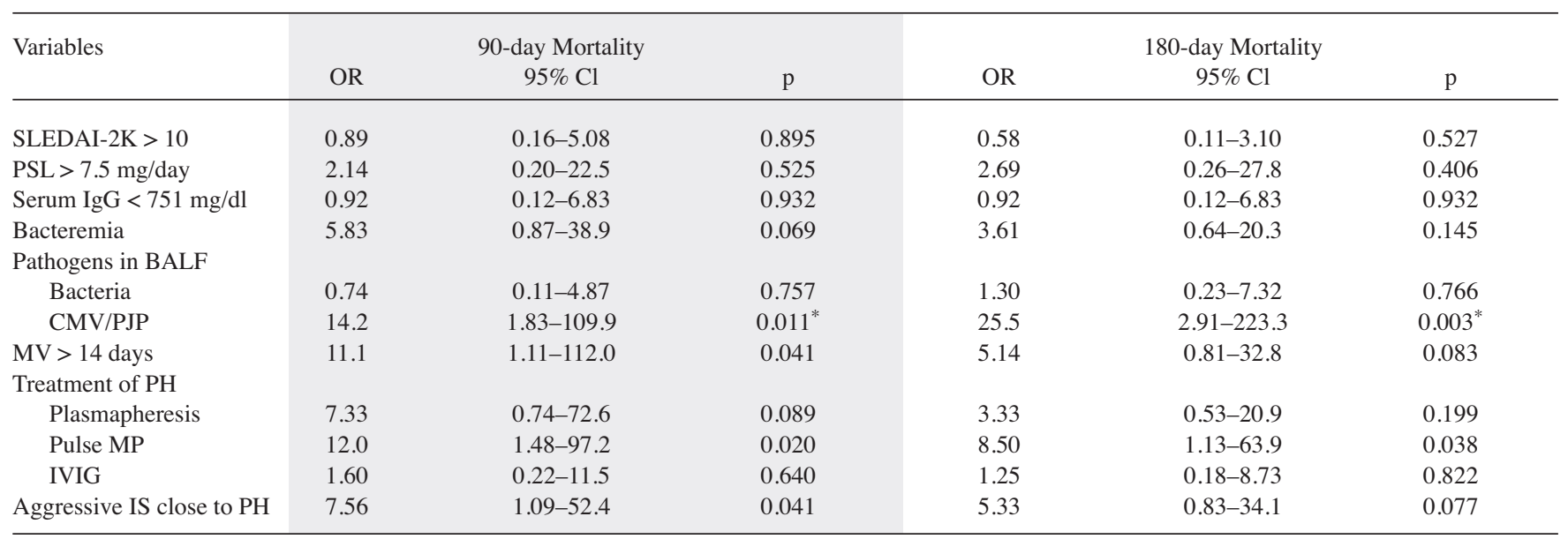

*The only factor that remained significant when selecting variables with $\mathrm{p}<0.05$ in multivariable analysis $(90$-day OR: $14.2,95 \% \mathrm{Cl} 1.83-109.9 ; \mathrm{p}=0.011$; 180-day OR: 25.5, 95\% Cl 2.91-223.3, p = 0.003). SLE: systemic lupus erythematosus; BALF: bronchoalveolar lavage fluid; CMV/PJP: cytomegalovirus or Pneumocystic jiroveci pneumonia; IVIG: intravenous Ig; IS: immunosuppression; MV: mechanical ventilation; MP: methylprednisolone; PSL: prednisolone; PH: pulmonary hemorrhage; SLEDAI-2K: SLE Disease Activity Index 2000.

patients with SLE (OR 11.1, 95\% CI 1.11-112, and OR 7.56, 95\% CI 1.09-52.4, respectively; Table 3). Baseline SLE activity, prednisolone dose equivalent to $>7.5 \mathrm{mg} / \mathrm{day}$, hypogammaglobulinemia, presence of bacteremia during $\mathrm{PH}$, presence of bacteria in BALF, and use of plasmapheresis for PH treatment did not alter survival status. Multivariable analysis indicated that CMV/PJP was associated with short- and longterm mortality rates in SLE patients with PH and were statistically significant.

Aggressive immunosuppression close to PH onset. Seven SLE patients received aggressive immunosuppression close to $\mathrm{PH}$ onset (Table 4). Seven patients received pulse methylprednisolone within 30 days before $(n=3)$ or after PH $(n=4)$. Two patients received RTX within 180 days before PH. Six patients required mechanical ventilatory support for $\mathrm{PH}$.

Pneumonia or bloodstream infections were common in patients with SLE who received aggressive immunosuppression close to $\mathrm{PH}$ onset. Four patients had CMV/PJP identified from BALF, and 4 patients had bacteremia, CMV viremia, or candidemia. The mortality rate was extremely high for patients who received aggressive immunosuppression close to $\mathrm{PH}$ onset, with 3 (patients 1 to 3 ) dying by 90 days, and 4 patients (patients 1 to 4 ) dying within 180 days after PH (Table 4). Four of the 5 patients with presence of $\mathrm{CMV} / \mathrm{PJP}$ or bloodstream infections died. On the other hand, both patients without pathogens in BALF or bloodstream infections (patients 6 and 7) survived the PH (Table 4 and Supplementary Figure 2, available with the online version of this article).

\section{DISCUSSION}

In patients with SLE, the mortality rate for $\mathrm{PH}$ remains high but greatly depends on the status of BALF-confirmed 
Table 4. Outcomes of selected SLE patients with aggressive immunosuppression close to PH onset.

\begin{tabular}{|c|c|c|c|c|c|c|c|}
\hline Variables & 1 & 2 & 3 & 4 & 5 & 6 & 7 \\
\hline Admission age, yrs & 34.1 & 57.8 & 34.3 & 43.7 & 33.2 & 27.4 & 33.2 \\
\hline Sex & $\mathrm{F}$ & $\mathrm{F}$ & $\mathrm{F}$ & $\mathrm{F}$ & $\mathrm{F}$ & $\mathrm{F}$ & $\mathrm{F}$ \\
\hline SLEDAI-2K & 11 & 3 & 21 & 20 & 21 & 20 & 7 \\
\hline \multicolumn{8}{|l|}{ Aggressive immunosuppression } \\
\hline Pulse MP, days before $\mathrm{PH}$ & - & - & - &,+ 13 & - &,+ 3 &,+ 28 \\
\hline RTX, days before PH & - &,+ 68 & - &,+ 140 & - & - & - \\
\hline Pulse MP, days after PH &,+ 11 & - &,+ 9 &,+ 0 &,+ 6 & - & - \\
\hline Intensive care & + & + & + & + & + & + & - \\
\hline ETT+MV/ECMO & $+/-$ & $+/-$ & $+/-$ & $+/+$ & $+/-$ & $+/-$ & $-1-$ \\
\hline APACHE II & 27 & 32 & 24 & 26 & 24 & 26 & NA \\
\hline SOFA & 12 & 13 & 8 & 9 & 6 & 11 & NA \\
\hline \multicolumn{8}{|l|}{ Pathogens in BALF } \\
\hline CMV/PJP & $+1-$ & $+/+$ & $+/+$ & - & $+/-$ & - & - \\
\hline Bacteria & $\mathrm{SM}, \mathrm{AB}$ & - & - & - & - & - & - \\
\hline Bloodstream infection & SM & $\mathrm{SM}, \mathrm{AB}$ & CMV & $\mathrm{BC}, \mathrm{CA}$ & - & - & - \\
\hline 90-day mortality & + & + & + & - & - & - & - \\
\hline 180-day mortality & + & + & + & + & - & - & - \\
\hline
\end{tabular}

SLE: systemic lupus erythematosus; AB: Acinetobacter baumanii; APACHE II: Acute Physiology and Chronic Health Evaluation II; BALF: bronchoalveolar lavage fluid: BC: Bacillus cereus; CA: Candida albicans; CMV: cytomegalovirus pneumonia; ECMO: extracorporeal membrane oxygenation; ETT+MV: endotracheal intubation + mechanical ventilation; NA: not applicable; MP: methylprednisolone; PH: pulmonary hemorrhage; PJP: Pneumocystic jiroveci pneumonia; RTX: rituximab; SLEDAI-2K: SLE Disease Activity Index 2000; SM: Stenotrophomonas maltophilia; SOFA: sequential organ failure assessment.

CMV/PJP. SLE patients with CMV/PJP had poorer outcomes of $\mathrm{PH}$ than patients without these infections. Other risk factors for 90-day mortality were prolonged duration of mechanical ventilation (> 14 days) and use of aggressive immunosuppression close to $\mathrm{PH}$ onset, the latter of which was associated with risk of CMV/PJP and bloodstream infections. Use of pulse methylprednisolone for $\mathrm{PH}$ treatment increased short- and longterm mortality rates. These findings reinforce the role of BALF analysis for risk stratification in SLE patients with $\mathrm{PH}$.

In our study, all patients with pathogens from BALF received antibiotic or antivirus treatment, but a higher mortality rate was still noted in CMV/PJP-positive patients. We reviewed the published studies of SLE with PH mainly within the last 10 years and classified the studies according to the frequency of BAL procedures: (1) BAL procedures for all patients; (2) BAL procedures for some patients; and (3) no BAL procedure (Supplementary Table 1, available with the online version of this article). Infections commonly occurred during episodes of $\mathrm{PH}(23.7-39.3 \%$ of $\mathrm{PH}$ episodes). In the first group of studies in which BAL procedures were universally performed, additional pathogens were found in BALF analysis but not in other groups. This advantage may further reduce the mortality rate by early diagnosis and pathogen-specific treatment. Notably, the mortality rate (25.8\% of patients) was numerically lower in the first group than in the others (40.5-59.3\% of patients). Comparable data can be observed in our study (180-day mortality for all patients: $8 / 27,29.6 \%$ ). On the other hand, aggressive immunosuppression for $\mathrm{PH}$ was not universally required (34.6-63.6\% of $\mathrm{PH}$ episodes were treated with CYC, and 18.6-81.8\% of PH episodes were treated with pulse methylprednisolone therapy). In our present study, SLE patients without pathogens in BALF or disseminated infections benefited from appropriate immunosuppression with or without plasmapheresis for $\mathrm{PH}$, but patients with CMV/PJP in BALF had high risk of mortality despite receiving antimicrobial treatment. In addition, our data (Table 4 and Supplementary Figure 2, available with the online version of this article) indicated a poor outcome when using aggressive immunosuppression close to $\mathrm{PH}$ in patients with CMV/PJP or bloodstream infections. In the clinical situations with concurrent infections during $\mathrm{PH}$, less intensive immunosuppression in combination with pathogen-specific treatment also works well in many studies and ours.

Based on the above findings, we propose an algorithm of pathogen surveillance for SLE patients with episodes of PH (Figure 2); it includes BALF analysis and other procedures. First, survey the concurrent infections with BAL and other procedures (including CMV PCR from BALF and blood). Patients usually benefit from consultations with infectious disease specialists to optimize antibiotic treatment according to pathogens and host status. Second, use less intensive immunosuppression with or without plasmapheresis for $\mathrm{PH}$ if pathogen-free status is not confirmed. Then, adjust the intensity of immunosuppression according to the severity of ongoing PH and status of concurrent infections. Finally, if the patient is confirmed as being pathogen free or if the infection is under control, intensive immunosuppression can be used safely to control PH (Figure 2).

Personal non-commercial use only. The Journal of Rheumatology Copyright $@$ 2019. All rights reserved 


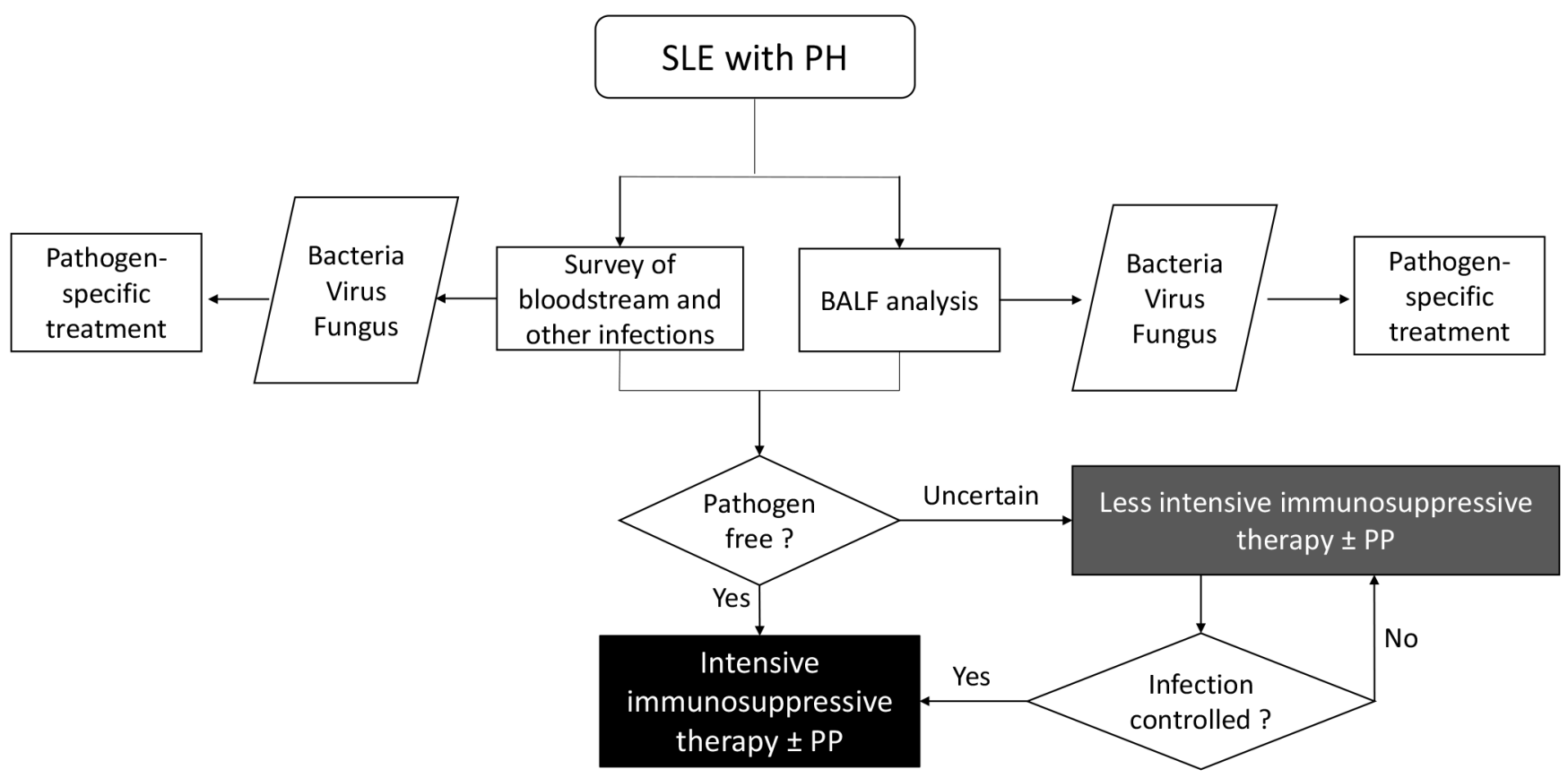

Figure 2. Proposed algorithm for management of PH in patients with SLE. SLE: systemic lupus erythematosus; BALF: bronchoalveolar lavage fluid analysis; PH: pulmonary hemorrhage; PP: plasmapheresis.

Infection rates in SLE patients with PH are quite high, ranging from $38.6 \%$ to $73.9 \%$ in different studies $1,5,6,7$. These rates are comparable to the $55.6 \%$ prevalence of pneumonia identified by BALF analysis here. Common pathogens included bacteria, Strongyloides stercoralis, and Aspergillus and other fungi ${ }^{1,5,6,7}$. CMV/PJP was more frequent $(8 / 27)$ in our study than in previous investigations ( $\leq 2$ cases/ study) $1,6,7,19,26$. The increased rate in our study may be secondary to immunosuppressive treatment for the high disease activity of SLE (median SLEDAI score for all patients: 12), or it may be due to the enhanced sensitivity of pathogen identification. This possibility highlights the value of universal BALF analysis for all suitable SLE patients with $\mathrm{PH}$.

The survival rate of SLE patients with PH is low but has improved in recent decades $(25-67 \%)^{4}$. The mortality rate may be even higher for SLE patients who are found to have CMV/PJP during PH. In our study, the 180 -day mortality rate was $75 \%$ for SLE patients with CMV/PJP and $10.5 \%$ for patients without these infections. Risk factors for mortality of PH in patients with SLE included higher APACHE II score, organ system failure score, nosocomial infection, mechanical ventilator usage, thrombocytopenia, renal failure, and septic shock $1,2,3,6,7,9,11,27$. In addition to these factors, our study revealed that CMV/PJP and pulse methylprednisolone for treatment of $\mathrm{PH}$ were associated with increased 90- and 180-day mortality rates, and that aggressive immunosuppression was associated with an increased 90-day mortality rate in SLE patients with PH.

In patients with SLE, PH may be treated by pulse methyl- prednisolone, CYC, plasmapheresis, or intravenous Ig (IVIG) treatment $4,6,10,11,19,20$. Pulse methylprednisolone is a standard treatment for PH but does not guarantee survival ${ }^{4,6,10,11,19}$. Reported mortality rates of $\mathrm{PH}$ ranged from $28.6 \%$ to $68.2 \%$ for patients with SLE who received pulse methylprednisolone (500-1000 mg/day), even after excluding pulmonary infections with BAL procedures in partial cases ${ }^{6,10,11}$. Infectious diseases were the most common causes of death ${ }^{6,11}$. In our study, 4 of the 7 patients with SLE who received aggressive immunosuppression close to $\mathrm{PH}$ onset died, all of whom had either CMV/PJP or bloodstream infections. Only 1 patient (patient 5 in Table 4) with CMV pneumonia survived through 180 days after $\mathrm{PH}$ despite pulse methylprednisolone therapy for PH. These data suggest a high association of occult infections with $\mathrm{PH}$, underscoring the need for universal BALF analysis for each PH event. CYC showed some survival benefit in previous reports ${ }^{4,6}$. Less intensive immunosuppression with plasmapheresis may be considered for mild $\mathrm{PH}$ cases or patients with sepsis/septic shock. IVIG was used in refractory PH with progressive infections ${ }^{6,11}$. In our study, IVIG was administered more frequently in SLE patients with CMV/PJP, which may be associated with these difficult-to-treat conditions. Further studies are required to develop an evidence-based strategy for patients with SLE with PH.

There are several limitations in our study, including the retrospective design, relatively small sample size, time gap between BAL procedure and PH onset, and lack of PCR confirmation of PJP. There may not be enough power to identify additional risk factors for PH-related mortality. The sensitivity of PJP diagnosed by microscopy would be lower

Personal non-commercial use only. The Journal of Rheumatology Copyright (C) 2019. All rights reserved. 
than PCR. This drawback suggests the underestimation of PJP in our SLE patients with PH. BALF analysis is an invasive procedure that is not suitable for patients with high oxygen demand without mechanical ventilatory support or extracorporeal membrane oxygenation. Some patients did not undergo a BAL procedure because of critical illness, a circumstance that might contribute to selection bias.

Pulmonary infections, especially CMV/PJP, are highly associated with $\mathrm{PH}$ and with increased mortality in SLE patients with PH. For SLE patients with PH, we recommend the use of BALF analysis for risk stratification and pathogen identification and the cautious use of pulse methylprednisolone or aggressive immunosuppression to reduce the risk of opportunistic infection.

\section{ACKNOWLEDGMENT}

The authors acknowledge the Department of Information Management of Taipei Veterans General Hospital for assistance in data acquisition.

\section{ONLINE SUPPLEMENT}

Supplementary material accompanies the online version of this article.

\section{REFERENCES}

1. Rojas-Serrano J, Pedroza J, Regalado J, Robledo J, Reyes E, Sifuentes-Osornio J, et al. High prevalence of infections in patients with systemic lupus erythematosus and pulmonary haemorrhage. Lupus 2008;17:295-9.

2. Kwok SK, Moon SJ, Ju JH, Park KS, Kim WU, Cho CS, et al. Diffuse alveolar hemorrhage in systemic lupus erythematosus: risk factors and clinical outcome: results from affiliated hospitals of Catholic University of Korea. Lupus 2011;20:102-7.

3. Kim D, Choi J, Cho SK, Choi CB, Kim TH, Jun JB, et al. Clinical characteristics and outcomes of diffuse alveolar hemorrhage in patients with systemic lupus erythematosus. Semin Arthritis Rheum 2017;46:782-7.

4. Ednalino C, Yip J, Carsons SE. Systematic review of diffuse alveolar hemorrhage in systemic lupus erythematosus: focus on outcome and therapy. J Clin Rheumatol 2015;21:305-10.

5. Quadrelli SA, Alvarez C, Arce SC, Paz L, Sarano J, Sobrino EM, et al. Pulmonary involvement of systemic lupus erythematosus: analysis of 90 necropsies. Lupus 2009;18:1053-60.

6. Martinez-Martinez MU, Abud-Mendoza C. Predictors of mortality in diffuse alveolar haemorrhage associated with systemic lupus erythematosus. Lupus 2011;20:568-74.

7. Martinez-Martinez MU, Sturbaum AK, Alcocer-Varela J, Merayo-Chalico J, Gómez-Martin D, Gómez-Bañuelos Jde J, et al Factors associated with mortality and infections in patients with systemic lupus erythematosus with diffuse alveolar hemorrhage. J Rheumatol 2014;41:1656-61.

8. Escuissato DL, Warszawiak D, Marchiori E. Differential diagnosis of diffuse alveolar haemorrhage in immunocompromised patients. Curr Opin Infect Dis 2015;28:337-42.

9. Chang MY, Fang JT, Chen YC, Huang CC. Diffuse alveolar hemorrhage in systemic lupus erythematosus: a single center retrospective study in Taiwan. Ren Fail 2002;24:791-802.

10. Andrade C, Mendonça T, Farinha F, Correia J, Marinho A, Almeida I, et al. Alveolar hemorrhage in systemic lupus erythematosus: a cohort review. Lupus 2016;25:75-80.
11. Shen M, Zeng X, Tian X, Zhang F, Zeng X, Zhang X, et al. Diffuse alveolar hemorrhage in systemic lupus erythematosus: a retrospective study in China. Lupus 2010;19:1326-30.

12. Araujo DB, Borba EF, Silva CA, Campos LM, Pereira RM, Bonfa $\mathrm{E}$, et al. Alveolar hemorrhage: distinct features of juvenile and adult onset systemic lupus erythematosus. Lupus 2012;21:872-7.

13. Chen M, Tian X, Qin F, Zhou J, Liu J, Wang M, et al. Pneumocystis pneumonia in patients with autoimmune diseases: a retrospective study focused on clinical characteristics and prognostic factors related to death. PLoS One 2015;10:e0139144.

14. Agustí C, Ramirez J, Picado C, Xaubet A, Carreras E, Ballester E, et al. Diffuse alveolar hemorrhage in allogeneic bone marrow transplantation. A postmortem study. Am J Respir Crit Care Med 1995;151:1006-10.

15. Vincent B, Flahault A, Antoine M, Wislez M, Parrot A, Mayaud C, et al. AIDS-related alveolar hemorrhage: a prospective study of 273 BAL procedures. Chest 2001;120:1078-84.

16. Chan CN, Lai FM, Lai KN, Pang JA. Relapse of idiopathic pulmonary haemorrhage and glomerulonephritis associated with cytomegalovirus (CMV) infection. Postgrad Med J 1988;64:52-5.

17. Ljungman P, Griffiths P, Paya C. Definitions of cytomegalovirus infection and disease in transplant recipients. Clin Infect Dis 2002;34:1094-7.

18. Reid AB, Chen SC, Worth LJ. Pneumocystis jirovecii pneumonia in non-HIV-infected patients: new risks and diagnostic tools. Curr Opin Infect Dis 2011;24:534-44.

19. Santos-Ocampo AS, Mandell BF, Fessler BJ. Alveolar hemorrhage in systemic lupus erythematosus: presentation and management. Chest 2000;118:1083-90.

20. Tse JR, Schwab KE, McMahon M, Simon W. Rituximab: an emerging treatment for recurrent diffuse alveolar hemorrhage in systemic lupus erythematosus. Lupus 2015;24:756-9.

21. Hochberg MC. Updating the American College of Rheumatology revised criteria for the classification of systemic lupus erythematosus. Arthritis Rheum 1997;40:1725.

22. Petri M, Orbai AM, Alarcón GS, Gordon C, Merrill JT, Fortin PR, et al. Derivation and validation of the Systemic Lupus International Collaborating Clinics classification criteria for systemic lupus erythematosus. Arthritis Rheum 2012;64:2677-86.

23. Knaus WA, Draper EA, Wagner DP, Zimmerman JE. APACHE II: a severity of disease classification system. Crit Care Med 1985;13:818-29.

24. Vincent JL, Moreno R, Takala J, Willatts S, De Mendonca A, Bruining $\mathrm{H}$, et al. The SOFA (Sepsis-related Organ Failure Assessment) score to describe organ dysfunction/failure. On behalf of the Working Group on Sepsis-Related Problems of the European Society of Intensive Care Medicine. Intensive Care Med 1996;22:707-10

25. Lim CC, Liu PY, Tan HZ, Lee P, Chin YM, Mok IY, et al. Severe infections in patients with lupus nephritis treated with immunosuppressants: A retrospective cohort study. Nephrology 2017;22:478-84

26. Zamora MR, Warner ML, Tuder R, Schwarz MI. Diffuse alveolar hemorrhage and systemic lupus erythematosus. Clinical presentation, histology, survival, and outcome. Medicine 1997;76:192-202.

27. Fatemi A, Shamsaee S, Raeisi A, Sayedbonakdar Z, Smiley A. Prognostic factors of mortality in Iranian patients with systemic lupus erythematosus admitted to intensive care unit. Clin Rheumatol 2017;36:2471-7 